\title{
A mudança de modelo assistencial de cuidado ao idoso na Saúde Suplementar: identificação de seus pontos-chave e obstáculos para implementação
}

\section{$\mathrm{I}^{1}$ Martha Regina de Oliveira, ${ }^{2}$ Renato Peixoto Veras, ${ }^{3}$ Hesio de Albuquerque}

\section{Cordeiro, ${ }^{4}$ Maria Teresa Pasinato I}

Resumo: A partir de uma análise crítica sobre os atuais modelos de atenção à saúde para idosos, este artigo apresenta uma proposta de linha do cuidado para este segmento etário, tendo como foco a promoção e a prevenção da saúde, de modo a evitar a sobrecarga do sistema de saúde. Enfatiza-se o setor suplementar, pois como um quarto da população utiliza este sistema, uma discussão mais minuciosa se faz necessária. $O$ conhecimento científico já identificou corretamente os fatores de risco para a população idosa, mas isso não basta. É prioritário utilizar esse conhecimento para efetuar a necessária transição do modelo assistencial clínico para o preventivo. Este precisa se configurar como um fluxo de ações de educação, promoção da saúde, prevenção de doenças evitáveis, postergação de moléstias, cuidado precoce e reabilitação de agravos. Não se nega a importância das instâncias pesadas (hospital, instituições de longa permanência, entre outras), mas estamos particularmente preocupados com uma abordagem contemporânea, que cuide de forma adequada o idoso e diminua custos. Se não for deste modo, o sistema se torna inviável.

> Palavras-chave: envelhecimento humano; idoso; prevenção de doenças; doença crônica; percurso assistencial; linha de cuidado.

\author{
1 Agência Nacional de Saúde \\ Suplementar. Rio de Janeiro-RJ, \\ Brasil (martha.oliveira@ans. \\ gov.br). \\ ${ }^{2}$ Universidade do Estado do \\ Rio de Janeiro, Universidade \\ Aberta da Terceira Idade. Rio de \\ Janeiro-RJ, Brasil (unativeras@ \\ gmail.com) \\ ${ }^{3}$ Universidade do Estado do Rio \\ de Janeiro, Universidade Aberta \\ da Terceira Idade. Rio de Janeiro- \\ RJ,Brasil (hesioalbuquerque@ \\ gmail.com). \\ ${ }^{4}$ Agência Nacional de Saúde \\ Suplementar. Rio de Janeiro-RJ, \\ Brasil (mtpasinato@gmail.com).
}

Recebido em: 03/09/2015 Aprovado em: 06/02/2016 
O envelhecimento populacional é um fenômeno mundial, largamente reconhecido, e o aumento da expectativa de vida pode ser entendido como uma das principais conquistas das sociedades nas últimas décadas. Menos de 10\% das pessoas de 65 anos ou mais estão livres de algum tipo de agravo crônico à saúde, e mais de $10 \%$ referem pelo menos cinco doenças crônicas concomitantes (BANCO MUNDIAL, 2011).

Segundo a Organização Mundial da Saúde (OMS), a nova realidade demográfica e epidemiológica brasileira aponta para a urgência de mudanças nos paradigmas de atenção à saúde da população idosa (KALACHE; KICKBUSCH, 1997), com estruturas criativas e inovadoras, acompanhadas de ações diferenciadas para que o idoso usufrua integralmente os anos proporcionados pelo avanço da ciência (OMS, 2008). É provável que os gastos em saúde aumentem substancialmente (CONNER, 2013). De fato, os cuidados em saúde tendem a emergir como um dos maiores desafios fiscais nas próximas décadas no Brasil. Existem duas forças por trás da projeção de aumento desses gastos: a proporção crescente de idosos na população e a intensidade do uso dos serviços de saúde pelos idosos (VERAS, 2011).

O envelhecimento da população gera novas demandas para os sistemas de saúde. Os idosos possuem particularidades bem conhecidas: mais doenças crônicas, mais custos, mais fragilidades, menos recursos sociais e financeiros; têm necessidades específicas, advindas das características clínico-funcionais e sócio-familiares peculiares a esse grupo etário. Os problemas de saúde dos idosos - maiores vítimas dos efeitos de sua própria fragilidade - desafiam os modelos tradicionais de cuidado (VERAS, 2013).

Como consequência de uma população mais envelhecida, a promoção e a educação em saúde, a prevenção e o retardamento de doenças e fragilidades, a manutenção da independência e da autonomia são ações que precisam ser ampliadas. Afinal, não basta simplesmente viver mais; é essencial que os anos adicionais sejam desfrutados com qualidade, dignidade e bem-estar (VERAS, 2015a). Urge, com isso, reformatar o modelo de assistência à saúde dos idosos, de forma a abarcar as diferenças nas condições de saúde desse contingente populacional, sem perder de vista suas especificidades e peculiaridades (VERAS, 2008). 
O objetivo da assistência à saúde em uma sociedade que envelhece é aumentar a qualidade de vida dos idosos através do aumento do tempo vivido com saúde e do envelhecimento saudável. Com isso, o impacto do envelhecimento nos sistemas de saúde é um assunto que ocupa grande espaço nas discussóes e preocupações de gestores em todos os países, especialmente no que contempla questôes como a promoção da saúde e prevenção dos riscos e doenças (PORTER; TEISBERG, 2009). Essa discussão é primordial também no setor de saúde suplementar brasileira, uma vez que afeta diretamente a sustentabilidade econômico-financeira e assistencial do setor (BONFADA, 2015). Para que o envelhecimento deixe de ser um "problema" e se torne uma "oportunidade", como propõem alguns estudiosos do tema, é preciso reformatar com urgência o modelo assistencial vigente, com um cuidado diferenciado, sobretudo para o idoso (LIMA-COSTA; VERAS, 2003). O cuidado com a saúde dos idosos inclui o tratamento psicológico e físico dos problemas rotineiros dos idosos com o objetivo de prevenir as doenças e incapacidades, além de promover o bem-estar dos indivíduos sempre que possível (MORAES, 2012).

Este artigo de opinião se propõe apresentar um breve retrospecto da assistência à saúde do idoso no setor suplementar, além das medidas adotadas nos últimos dez anos para a mudança do modelo vigente. A seguir serão apresentadas as questôes basilares para a estruturação de um novo modelo de cuidado ao idoso na saúde suplementar. Os principais obstáculos a serem enfrentados são detalhados em um segmento específico. Por fim, são traçados alguns comentários sobre o imperativo de se buscar a reformatação da assistência à saúde dos idosos no setor suplementar, sob pena de não se avançar na qualidade da assistência à saúde prestada, com importantes prejuízos para o bem-estar da população atendida por planos privados de atenção à saúde.

\section{Breve retrospecto do modelo de assistência à saúde do idoso no setor suplementar}

O modelo assistencial hegemônico na saúde suplementar caracteriza-se pelo enfoque biologicista da saúde/doença, desconsiderando seus determinantes sociais, com ações desarticuladas, desintegradas, pouco cuidadoras, centradas na assistência médico-hospitalar especializada e com incorporação acrítica de novas tecnologias, constituindo um modelo pouco eficiente, pouco eficaz e pouco 
efetivo (BRASIL, 2004). Nesse modelo assistencial, os indivíduos são atendidos a partir da demanda espontânea, suscitada pela presença de sintomas ou doenças. Há concentração crescente de consultas médicas especializadas, exames diagnósticos, terapias, internaçoos e cirurgias, que nem sempre se traduzem em maior resolutividade e recuperação da saúde (HERNANDES, 2012).

As demandas na área de saúde dos idosos requerem análise especial, já que seus padrôes de morbimortalidade diferem dos observados para a população jovem e se caracterizam por enfermidades crônicas, cujos custos diretos e indiretos são mais elevados. Muito idosos apresentam múltiplas patologias e frequentam inúmeros especialistas, tornando a questão da iatrogenia um problema de saúde para o próprio indivíduo e para o sistema, com consequências indesejáveis para ambos (VERAS, 2003).

Evidências demonstram que, para que o modelo de atenção à saúde do idoso seja eficiente, é necessário que ele compreenda atividades organizadas em um fluxo hierarquizado, de forma que mesmo sendo as atividades executadas de forma independente, elas se inter-relacionem. $\mathrm{O}$ atendimento ao idoso de forma fragmentada, sem avaliação da sua funcionalidade e sem a compreensão das repercussões do envelhecimento nos processos saúde-doença, repercute negativamente na sua saúde (VERAS, 2013).

Em função dessa situação, a Agência Nacional de Saúde Suplementar (ANS) vem estudando e propondo ações que visam mudar essa lógica de prestação de serviços através da introdução de um novo modelo de assistência, que seja mais efetivo nos resultados assistenciais e nos custos.

Nos últimos dez anos, a ANS avançou nas políticas indutoras, buscando um modelo de assistência à saúde menos fragmentado e mais preocupado com a atenção às multipatologias que são característicos do processo de envelhecimento (VERAS, 2013). A primeira iniciativa empreendida pela Agência consistiu na publicação, em março de 2005, da Resolução Normativa (RN) no 94, estabelecendo critérios para a prorrogação dos prazos para a integralização da cobertura com ativos garantidores das provisões de risco, pelas operadoras de planos privados de assistência à saúde que desenvolvessem programas de promoção da saúde e prevenção de riscos e doenças.

Em 2008, a ANS aprovou novos incentivos para estimular o desenvolvimento de programas de promoção da saúde e prevenção de riscos e doenças, por meio da 
Instrução Normativa (IN) conjunta no 01 DIPRO/DIOPE. De acordo com essa norma, as operadoras de planos privados de assistência à saúde que desenvolvam e cadastrem os mesmos na ANS passam a contar com a possibilidade de contabilizar os valores neles aplicados como investimentos no Plano de Contas Padrão, que deve ser enviado periodicamente à ANS. O entendimento da Agência foi que os recursos utilizados nesses programas consistem em investimentos, pois podem gerar benefícios futuros às operadoras e a seus beneficiários (ANS, 2009).

Tendo em vista o acelerado processo de envelhecimento da população brasileira, a ANS constituiu, em setembro de 2010, um grupo de trabalho voltado para a incorporação do conceito de Envelhecimento Ativo, como definido pela OMS, em consonância com as políticas empreendidas pelo Ministério da Saúde (MS), ressalvando, no entanto, as diferenças e particularidades existentes para o setor suplementar da saúde no Brasil. A proposta, baseada no conceito de envelhecimento ativo, consiste em buscar melhorias na qualidade de vida e manutenção da capacidade funcional de toda a população, na medida em que a mesma envelhece. É grande o desafio enfrentado pela ANS, no sentido de induzir uma reorganização no setor de saúde suplementar para um modelo de atenção à saúde mais voltada para o cuidado integrado das condições crônicas, que leve à racionalização dos recursos e produza melhoria da saúde. Essa não é uma questão fácil, pois envolve mudanças culturais e comportamentais dos vários segmentos sociais que atuam na saúde suplementar - beneficiários, operadoras e prestadores.

Em 2011, foi aprovada a $R N n^{\circ}$ 265/2011, que estabeleceu a opção por oferta da bonificação e premiação para beneficiários que aderissem a programas de promoção do envelhecimento ativo, voltados para todas as faixas etárias. A estratégia foi testada e utilizada em outros países com a capacidade de aumentar a adesão a esses programas (ANS, 2011a; 2011b). Em 2012, a ANS propôs o conceito de plano de cuidado do idoso na saúde suplementar, que engloba os conceitos de linha de cuidado e projeto terapêutico (MALTA; MERHY, 2010). Assim, o plano de cuidado engloba a avaliação do beneficiário desde sua entrada no sistema, com a estratificação do seu risco, avaliação do estado funcional e definição da melhor linha de cuidado a ser seguida, como o caminho a ser percorrido a partir de então. Esse caminho inclui procedimentos fundamentais para seu sucesso, tais como hierarquização de rede, programas de promoção da saúde e prevenção dos riscos e doenças, além de todas as açôes articuladas e integrados aos cuidados. 
Essas propostas apresentam, em seu conjunto, esforços para reorientar o modelo assistencial vigente. Mas interessa-nos saber quais pontos seriam pilares importantes dentro desta concepção e quais seriam as dificuldades a serem enfrentadas e que pudessem ser obstáculos para sua implementação.

\section{Os pilares da nova estrutura proposta para o cuidado ao idoso na saúde suplementar}

Uma importante característica do contingente idoso é sua heterogeneidade. O mesmo subgrupo populacional é composto tanto por indivíduos hígidos e autônomos, quanto por aqueles com dificuldades para realizar as atividades básicas do cotidiano, como tomar banho sozinho ou alimentar-se. A mudança do modelo de assistência à saúde dos idosos passa, dessa forma, por formular novas concepçõos de atenção à saúde capazes de abarcar as diferenças nas condiçõos de saúde dos idosos que respeitem suas especificidades e peculiaridades. Ao estudarmos as propostas da ANS, ressaltamos algumas questôes imprescindíveis para a efetivação do modelo:

1) Hierarquização do cuidado: fundamental para a organização da gestão dos pacientes e para que se possa utilizar o sistema de saúde em sua plenitude, de maneira mais ágil, sem duplicidade de cuidados e diminuindo a iatrogenia do sistema.

2) Organização da demanda baseada em identificação do perfil de risco: ação extremamente importante para conhecer a carteira dos usuários e de organizar a prestação de serviços focada nas suas necessidades e visando reduzir danos e riscos à saúde.

3) Busca ativa e informação ao paciente: proposta bem diferente da forma como se dá o cuidado hoje na saúde suplementar, visa promover a saúde e não somente tratar da doença no momento em que esta já se instalou, muitas vezes de forma grave. A informação ao paciente e sua família também é essencial para se promover o autocuidado e a consciência sanitária.

4) Reorientação do foco da ausência de doença pelo foco no cuidado da saúde: com o avançar da idade, é cada vez mais difícil que os indivíduos não apresentem doenças crônicas, o que não necessariamente implica deterioração da qualidade de vida dos mesmos, pois o cuidado adequado pode minimizar os problemas decorrentes dessas doenças. 
5) Busca de adesão e participação dos beneficiários através de incentivos:

proporciona corresponsabilização e maior fidelização aos projetos de cuidado integral à saúde.

Essas questões são interdependentes. Projetos voltados para a mudança do foco da ausência de doença pelo foco no cuidado da saúde, por exemplo, funcionam melhor quando articulados com projetos voltados para a busca ativa e informação ao paciente. Da mesma forma, é possível pensar a organização da demanda com base no perfil de risco, a partir das açôes voltadas para a busca ativa da população idosa (CALDAS et al., 2013).

\section{Os obstáculos a serem enfrentados}

A proposta também contém alguns obstáculos que precisam ser debatidos e vencidos, muitos deles em uma discussão com a sociedade. Dentre eles, destacamos:

1) A expectativa do usuário do direito ao consumo ao invés de cuidado. Esse é um dos pontos que merece destaque. Hoje, em nossa sociedade, a saúde se tornou um bem de consumo associado ao uso de alta tecnologia, sem nenhuma relação com o resultado assistencial que se venha ter. Ter acesso a um plano de saúde significa, no imaginário das pessoas, ter acesso ilimitado a um conjunto de procedimentos possíveis de serem realizados. Não há educação em saúde ou orientação aos indivíduos sobre autocuidado ou estratégias de promoção e prevenção. Ninguém espera comprar um plano de saúde para ter acesso a um cuidado organizado e planejado, mas ter acesso ilimitado, a seu bel prazer, na rede de serviços de saúde, na hora que que desejar. A inversão dessa lógica é de extrema relevância para o melhor funcionamento do modelo, mas se trata de assunto de difícil construção e multifacetado, que precisa ser enfrentado com urgência.

2) Necessidade de redesenhar a prestação de serviço, hoje orientada pela produção focada na alta complexidade, na fragmentação do cuidado através de múltiplas especialidades, totalmente desarticuladas, que não se falam e que não registram em um prontuário único as múltiplas opiniões, além dos inúmeros fármacos e exames prescritos. 
1390 3) Necessidade de ofertar serviços resolutivos, pautado em modelos onde haja hierarquização dos cuidados, em protocolos validados e na gestão focada na atenção básica.

4) Necessidade de capacitação profissional, voltada para uma lógica de atenção primária e pautada na busca ativa de casos e voltada para atender às necessidades integrais do indivíduo.

5) Medo, por parte das operadoras de planos de saúde, de possíveis "processos agregados", como a possibilidade de introdução de cuidador, homecare e assistência social à cobertura obrigatória ou aumento de judicialização dessas demandas.

6) Cultura da desconfiança na prestação de serviço entre os atores da saúde suplementar brasileira.

7) Necessidade de organização da gestão da operadora, hoje ainda muito focada na área financeira e não na assistencial.

8) Necessidade de mudança do modelo de alta produção de procedimentos como forma remuneratória, sem nexo com a produção de resultados assistenciais.

Em suma, este artigo aponta a necessidade de se rever a forma como se dá o cuidado ao idoso hoje na saúde suplementar brasileira, perpassando diferentes pontos a serem trabalhados. A reformatação desse modelo, pouco eficiente e com resultados assistenciais bastante aquém dos ideais, precisa ser tratada em diferentes esferas, sob diferentes óticas, tanto assistenciais, econômicas e socioculturais (VERAS et al., 2015b).

Sabemos que o idoso tem muitas doenças e, naturalmente, utiliza muito os serviços de saúde. Portanto, deveria-se oferecer um cuidado em instâncias mais leves, sempre que possível em caráter preventivo, preferencialmente fora do ambiente hospitalar. Envelhecer, ainda que sem doenças crônicas, envolve alguma perda funcional, que é expressa por diminuição de vigor, força, prontidão, velocidade de reação sistêmica e eficiência metabólica (VERAS; CALDAS; CORDEIRO, 2013).

\section{Considerações finais}

Apesar de difíceis, as mudanças apontadas precisam ser implementadas o mais breve possível. A prestação de serviços de saúde, atualmente, fragmenta a atenção 
ao idoso, com multiplicação de consultas de especialistas, inúmeros fármacos,

exames e outros procedimentos. Sobrecarrega o sistema, provoca forte impacto financeiro em todos os níveis e não gera benefícios significativos para a qualidade de vida (NERI et al., 2004).

Nossa proposta é que o modelo de atenção ao idoso tenha como foco a identificação de riscos potenciais. Ao monitorarmos a saúde em vez da doença, direcionamos o investimento dos recursos do sistema de saúde para uma intervenção precoce, que resulta em chances mais generosas de reabilitação e redução do impacto na funcionalidade (VERAS et al., 2014).

Qualquer política contemporânea para o setor deve valorizar o envelhecimento saudável, com manutenção e melhoria da capacidade funcional, prevenção de doenças e recuperação da saúde e das capacidades funcionais. Sem uma abordagem preventiva e integral, que associe reflexão epidemiológica e planejamento sistemático de ações de saúde, não há saída possível para a crise de financiamento e reestruturação do setor. ${ }^{1}$

\section{Referências}

AGÊNCIA NACIONAL DE SAÚDE SUPLEMENTAR (Brasil). Atenção à saúde no setor suplementar: evolução e avanços do processo regulatório. Rio de Janeiro: ANS, 2009.

. Resolução Normativa no 264, de 19 de agosto de 2011, da Diretoria de Normas e Habilitação de Produtos- DIPRO. Rio de Janeiro, 2011a.

Resolução Normativa no 265, de 19 de agosto de 2011, da Diretoria de Normas e Habilitação de Produtos- DIPRO. Rio de Janeiro, 2011b.

BANCO MUNDIAL. Population aging: is Latin America ready? Directions in development. Washington, DC: The World Bank, 2011.

BRASIL. Ministério da Saúde. Secretaria de Vigilância à Saúde. Saúde Brasil 2004: uma análise da situação de saúde. Brasília: Ministério da Saúde, 2004.

BONFADA, D. Gasto com a internação de idosos em unidades de terapia intensiva. 2015. 105 f. Tese de doutorado (Doutorado em Saúde Coletiva) - Universidade Federal do Rio Grande do Norte. Centro de Ciências da Saúde. Programa de Pós-Graduação em Saúde Coletiva, Natal/RN, 2015.

CALDAS, C. P. et al. Rastreamento do risco de perda funcional: uma estratégia fundamental para a organização da Rede de Atenção ao Idoso. Ciênc. saúde coletiva, v. 18, n. 12, p. 3495 3506, dez. 2013. 
CONNER, D. A. Quality and cost evaluation of a medical financial assistance program. The Permanent Journal, v. 17, n. 1, p. 31-37, 2013.

HERNANDES, E. S. et al. Idosos com e sem plano de saúde e características socioepidemiológicas associadas. Rev. Saúde Pública, v. 46, n. 6, p. 1030-1038, 2012. Disponível em: <http://dx.doi.org/10.1590/S0034-89102012000600013>. Acesso em: 02 out. 2015.

KALACHE, A.; KICKBUSCH, I. A global strategy for healthy ageing. World Health, v. 50, n. 4, p. 4-5, jul-ago 1997.

LIMA-COSTA, M. F.; VERAS, R. P. Saúde pública e envelhecimento. Cadernos Saúde Pública. Rio de Janeiro, v. 19, n. 3, jun. 2003. Disponível em: http://www.scielo.br/scielo. php?script=sci_arttext $\&$ pid $=$ S0102-311X2003000300001\&lng=en \&nrm $=$ iso. Acesso em: 02 out. 2015.

MALTA, D. C.; MERHY, E. E. O percurso da linha do cuidado sob a perspectiva das doenças crônicas não transmissíveis. Interface. Botucatu, v. 14, n. 34, p. 593-606, 2010.

MORAES, E. N. Atenção à saúde do idoso: aspectos conceituais. Brasília: OPAS, 2012.

NERI, M.et al. Inflação e os idosos brasileiros. In: CAMARANO, A. A. (Org.). Os novos Idosos brasileiros: muito além dos 60? Rio de Janeiro: IPEA, 2004.

ORGANIZAÇÃO MUNDIAL DA SAÚDE. Relatório Mundial de Saúde: atenção primária em saúde. Agora mais do que nunca. Brasília: OMS, 2008.

PORTER, M. E.; TEISBERG, E. O. Repensando a saúde: estratégias para melhorar a qualidade e reduzir os custos. Porto Alegre: Artmed, 2009.

VERAS, R. P. et al. A assistência suplementar de saúde e seus projetos de cuidado para com o idoso. Ciênc. saúde coletiva. Rio de Janeiro, v. 13, n. 4, p. 1119-1126, ago. 2008.

VERAS, R. P. baseada na capacidade funcional. Rev. Bras. Geriatr. Gerontol. Rio de Janeiro v. 16, n. 2 , p. $385-392,2013$.

VERAS, R. P. Integração e continuidade do cuidado em modelos de rede de atenção à saúde para idosos frágeis. Rev. Saúde Públ. São Paulo, v. 48, n. 2, p. 357-365, abr. 2014.

VERAS, R. P. A necessária gestão qualificada na área da saúde: decorrência da ampliação do segmento etário dos idosos. J. Bras. Economia Saúde. Rio de Janeiro, v. 3, n. 3, p. 31-39, 2011b. (Especial Gerenciamento de doenças crônicas).

VERAS, R. P. A urgente e imperiosa modificação no cuidado à saúde da pessoa idosa. Rev . Bras. Geriatr. Gerontol. Rio de Janeiro, v. 18, n. 1, p. 5-6, 2015a.

VERAS, R. P. Garantir a saúde e o bem-estar dos idosos: desafios de hoje e amanhã. Rev. bras. geriatr. gerontol., v. 18, n. 3, p. 73-474, jul.-set. 2015 b. 
VERAS, R. P.; CALDAS, C. P.; CORDEIRO, H. de A. Modelos de atenção à saúde do idoso: repensando o sentido da prevenção. Physis: Revista de Saúde Coletiva. Rio de Janeiro, v. 23, n. 4, p. 1189-1213, dez. 2013.

\section{Nota}

${ }^{1}$ M. R. de Oliveira, R. P. Veras, H. A. Cordeiro e M. T. Pasinato participaram igualmente da redação do artigo, revisão crítica do conteúdo intelectual e aprovaram a versão final a ser publicada. Não há conflito de interesses. 


\section{Abstract}

The change of care model to elderly care in Supplementary Health: identification of its key points and obstacles to implementation

From a critical analysis of current models of health care for the elderly, this article proposes a care line for this age group, focusing on the promotion and health prevention in order to avoid overloading the health system. It emphasizes the supplementary sector, since a quarter of the population uses this system, a more thorough discussion is needed. Scientific knowledge has correctly identified the risk factors for the elderly, but this is not enough. It is a priority to use this knowledge to make the necessary transition from the clinical care to preventive model. This needs to be configured as a stream of education initiatives, health promotion, prevention of preventable diseases, postponement of diseases, early care and rehabilitation of injuries. There is no denying the importance of heavy bodies (hospital, long-term care facilities, etc.), but we are particularly concerned about an approach that improves the quality of life and decrease costs. If not so, the system becomes impractical.

> Keywords: human aging; elderly; diseases' prevention; chronicle diseases; care pathway; care line. 